\title{
Aligning Simulation Models of Smallpox Outbreaks
}

\author{
Li-Chiou Chen ${ }^{1}$, Boris Kaminsky ${ }^{1}$, Tiffany Tummino ${ }^{2}$, \\ Kathleen M. Carley ${ }^{12}$, Elizabeth Casman ${ }^{2}$, Douglas \\ Fridsma $^{3}$, Alex Yahja ${ }^{12}$ \\ ${ }^{1}$ Institute for Software Research International, School of Computer Science \\ ${ }^{2}$ Department of Engineering and Public Policy \\ Carnegie Mellon University, Pittsburgh, Pennsylvania 15213 \\ \{lichiou, borisk, ttummino, carley, casman\}@andrew.cmu.edu \\ ${ }^{3}$ Center for Biomedical Informatics, School of Medicine, University of Pittsburgh \\ Pittsburgh, Pennsylvania 15213 \\ fridsmaecbmi.pitt.edu
}

\begin{abstract}
We aligned two fundamentally different models of smallpox transmission after a bioterrorist attack: A location-explicit multi-agent model (BioWar) and the conventional epidemiological box model, called a SIR model for Susceptible-Infected-Recovered. The purpose of this alignment is part of a greater validation process for BioWar. From this study we were able to contribute to the overall validation of the complex agent based model, showing that, at the minimum, the epidemiological curves produced by the two models were approximately equivalent, both in overall and the time course of infection and mortality. Subtle differences on the model results revealed the impact of heterogeneous mixing in the spread of smallpox. Based on this foundation, we will be able to further investigate the policy responses against the outbreaks of contagious diseases by improving heterogeneous properties of agents, which cannot be simulated in a SIR model.
\end{abstract}

\section{Introduction}

Numerical simulation models can be used to estimate the impact of large-scale biological attacks and to design or select appropriate response strategies. The "correctness" of the model is critical since the "wrong" model may lead to "wrong" decisions, but no model is perfect and few models can ever be considered thoroughly validated. Studies $[32,33]$ have agreed that it is often too costly and time-consuming to determine if a model is absolutely valid. Instead, evaluations are conducted until sufficient confidence is obtained that a model is valid for its intended application. We developed a methodology to align an agent-based model of biological attack simulations (BioWar) against the classical susceptible-infected-recovered (SIR) box model as part of the validation process. Our purpose is to verify whether the agent-based model can produce results that closely resemble those of the well accepted and venerable SIR model, thus giving BioWar a sort of reflected credibility from the SIR model. This is not sufficient validation, but it is a confidence building step in the much larger task of validating BioWar. 
Aligning the two types of model is challenging because of their radically different structures. We demonstrate an objective methodology for translating key parameters between models, for running the models in concert to supply aligned inputs during simulations, and for evaluating the agreement between the models.

BioWar is a multi-agent simulation tool of biological attacks. It combines computational models of social networks, disease models, demographically resolved agent models, spatial models, wind dispersion models, and a diagnostic model into a single integrated system that can simulate the impact of a bioterrorist attack on any city [7]. For this paper, we restrict the alignment to the smallpox simulation in BioWar. The SIR model and its variations have been widely used to model the spread of epidemics and to study immunization strategies $[1,2,4,13]$. The SIR model is a "populationbased" aggregated representation of disease transmission that assumes homogeneous mixing of individuals. In contrast, BioWar models the complex social interactions and heterogeneity of mixing absent in most SIR models.

Model alignment, also referred to as "docking," is the comparison of two computational models to see if they can produce equivalent results. Properly done, model alignment can uncover the differences and similarities between models and reveal the relationships between the different models' parameters, structures, and assumptions. The purpose of aligning BioWar with the conventional box model is to demonstrate a general equivalence, as part of a greater validation process for BioWar. The concept of model alignment was first proposed by Axtell et al. [3]. We have used this method previously in validating BioWar's anthrax simulation [10].

This paper is organized as follows. The next section provides background information on smallpox and the two models. Section 3 explains our methodology of model alignment. Section 4 discusses our findings and compares the two models based on the simulation results. Conclusions and discussion of future work follow.

\section{Two models of smallpox transmission}

Smallpox has several distinct stages, including incubation, prodrome (earlysymptoms), and fulminant (late-symptoms). The initial site of viral entry is usually the mucous membranes of the upper respiratory tract. Once a person is infected, the incubation stage usually lasts for about 12 to 14 days. During this period, an infected person experiences no symptoms and is not contagious. The first symptoms of disease include fever (typically high), head and body aches, and possibly vomiting. This prodromal stage lasts about 2 to 4 days. During this time infected persons are usually too sick for normal activity, and may be contagious, although infectivity is often negligible [14].

The fulminant stage begins with the onset of rash. The rash appears first on the tongue and inside the throat or mouth, then appears on the face and limbs, usually spreading across the body within 24 hours. An infected person is most contagious within the first 7 to 10 days after the dermal rash appears. The rashes become bumps on about the $3^{\text {rd }}$ day of the fulminant phase. The pox fill with liquid and acquire a distinctive shape with a depression in the middle by the $4^{\text {th }}$ day of the period. Most smallpox deaths occur on the $5^{\text {th }}$ or $6^{\text {th }}$ day after the onset of rash $[27,23,35]$. Over a 
period of about 5 days after the pox fill with liquid, they become firm, sharply raised pustules; over another 5 days, these pustules crust and scab. Within about 14 days of the appearance of the rash, most of the pustules will have formed scabs. Within about 3 weeks after the onset of the rash, all of the scabs fall off, though the scab material is infectious somewhat longer.

Transmission of smallpox from an infected person to an uninfected person usually requires face-to-face personal contact, inhalation of droplets formed by coughing or sneezing, or contact with infected body fluids or contaminated objects (e.g., bedding) [8]. While infection has occurred through the spread of the virus through the air in buildings or other enclosed areas, this type of transmission has been rare. Humans are the only known reservoir of the virus, and there has been no known transmission via animals or insects.

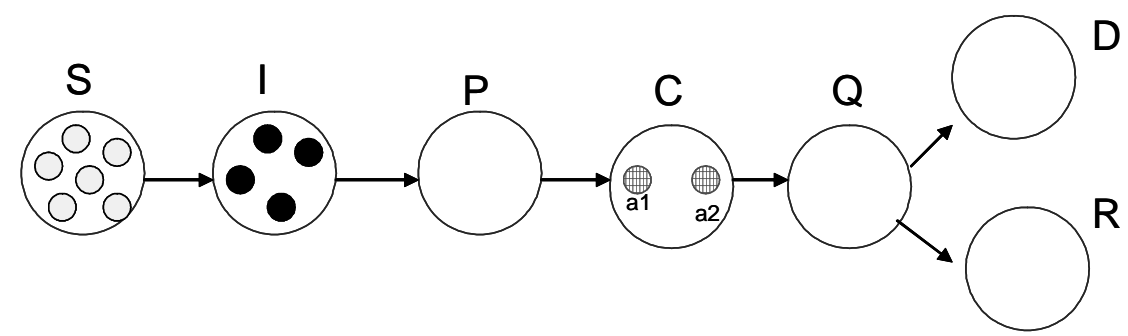

Fig 1a. An illustration of the SIR model. Individuals (represented as dots) in a state have the transition probability of moving to next state

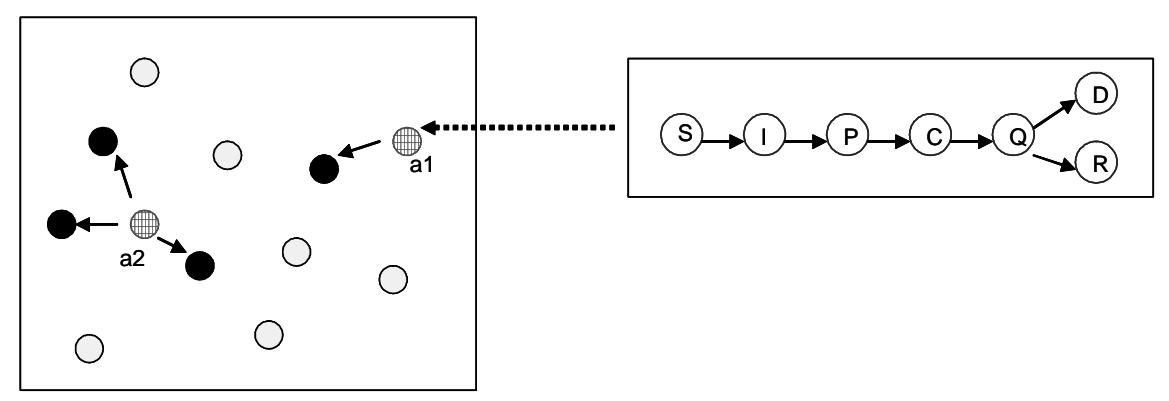

Fig 1b. An illustration of the disease transmission process in BioWar. Each individual (such as a1) has its own state machine and has a different reproductive rate (e.g. a1 infects one case but a2 infects 3 cases)

Two types of models have been used to study the progression of smallpox outbreaks. They are population-level box models $[6,17,24,26]$ and individual-level agent-based models [19]. These population-level models are either variations or stochastic versions of the basic SIR model. The SIR model $[1,2]$ is a widely used model of the spread of a disease through a population. As noted, the SIR model describes the epidemic diffusion process by categorizing the entire population into three states - susceptible, infectious and recovered - linked by differential equations. The SIR model 
assumes that the population is homogenous and completely mixed. All members of a particular state are identical and have predefined transition probabilities of moving to another state in the model (Fig. 1a).

In contrast, agent-based models assume a heterogonous population with mixing only within socially defined networks (Fig. 1b). BioWar models the residents of a city (agents) as they go about their lives. When a bioattack occurs, those in the vicinity of the release may become infected, following probabilistic rules based on received dose and age of the agent. The infected agents modify their behaviors as their disease progresses and they become unable to perform their normal functions. Susceptible agents are infected if they come within a certain distance with infectious agents following probabilistic rules concerning the likelihood of infection. BioWar is not just an agentbased model, but a network model where the networks vary dynamically based on agent activity. Agents interact within and through their social network which is based on their age, race, gender, socio-economic status, and job. Consequently, which agents are likely to be infected and to infect others, depends on things like time of day, location of attack, time of year, age of the agent, and so on. Unlike many other agent-based models, BioWar is tightly coupled to demographic, school, economic, and social data as well as physiological, disease, geographic, weather, and climate data. The fine grained detail by which heterogeneity is defined, and the level of detail in agents behaviors (e.g., they don't just get infected and die, they go to the hospital, to the doctor, to the pharmacy, and so on) means that BioWar, unlike SIR models, can be used to examine response behavior far beyond mortality indicies. A detailed description of the BioWar model is published in [7].

The mathematical equations of the modified SIR model used in this paper follow. This modified SIR model allows us to simulate the residual immunity in the population and vaccination or patient-isolation response strategies. As in (1), the total population $N$ is divided into seven states: susceptible $(S)$, incubation: infected but not yet infectious $(I)$, prodrome: infected with non-specific symptoms $(P)$, contagious with specific symptoms but not yet quarantined $(C)$, contagious with specific symptoms but quarantined $(Q)$, population that die $(D)$, and population that recover and become immune $(R)$.

$$
N=S+I+P+C+Q+D+R .
$$

Transition probabilities, $\beta, \sigma, \alpha, \gamma, v$, are the rates that the population changes from one state to another state, and $\lambda$ is the death rate.

We revised the original SIR model to cover different population groups so that it can be used to model residual immunity and vaccination. Let $g$ represent the number of population groups. For example, $g=1$ when the entire population is homogeneous as in our base scenario and $\mathrm{g}=3$ when we separate the population into three groups (no vaccination, residual immunity, vaccinated) as in our vaccination scenario. In this case, the population in each state is divided into these groups and the total population

$N$ is equal to $\sum_{i=1}^{g} N_{i}$. Each group has its own transition probability of reproduction $\beta$ and death rate $\lambda_{l}$. We assume that the disease-stage durations are the same across groups. Thus, transition probabilities, $\sigma, \alpha, \gamma, v$, are the same for each group. The differential equations of the SIR model are as (2) and (3). 


$$
\begin{aligned}
& \frac{d S_{i}}{d t}=-\beta_{i} S_{i} C, \quad \frac{d I_{i}}{d t}=\beta_{i} S_{i} C-\sigma I_{i}, \quad \frac{d P_{i}}{d t}=\sigma I_{i}-\alpha P_{i}, \quad \frac{d C_{i}}{d t}=\alpha P_{i}-\gamma C_{i}, \\
& \frac{d Q_{i}}{d t}=\gamma C_{i}-v Q_{i}, \quad \frac{d D_{i}}{d t}=\lambda_{i} v Q_{i}, \quad \frac{d R_{i}}{d t}=\left(1-\lambda_{i}\right) v Q_{i} . \\
& \frac{d S}{d t}=\sum_{i=1}^{g} \frac{d S_{i}}{d t}, \quad \frac{d I}{d t}=\sum_{i=1}^{g} \frac{d I_{i}}{d t}, \quad \frac{d P}{d t}=\sum_{i=1}^{g} \frac{d P_{i}}{d t}, \quad \frac{d C}{d t}=\sum_{i=1}^{g} \frac{d C_{i}}{d t} \\
& \frac{d Q}{d t}=\sum_{i=1}^{g} \frac{d Q}{d t}, \quad \frac{d D}{d t}=\sum_{i=1}^{g} \frac{d D_{i}}{d t}, \quad \frac{d R}{d t}=\sum_{i=1}^{g} \frac{d R_{i}}{d t} .
\end{aligned}
$$

\section{Model Alignment}

We first aligned the input parameters (Section 3.1) of the two models by calculating the reproductive rates from BioWar experiments (Section 3.2). We then designed scenarios to simulate smallpox outbreaks using the two models (Section 3.3), and compared population level results (Section 4). Fig. 2 illustrates our alignment methodology.

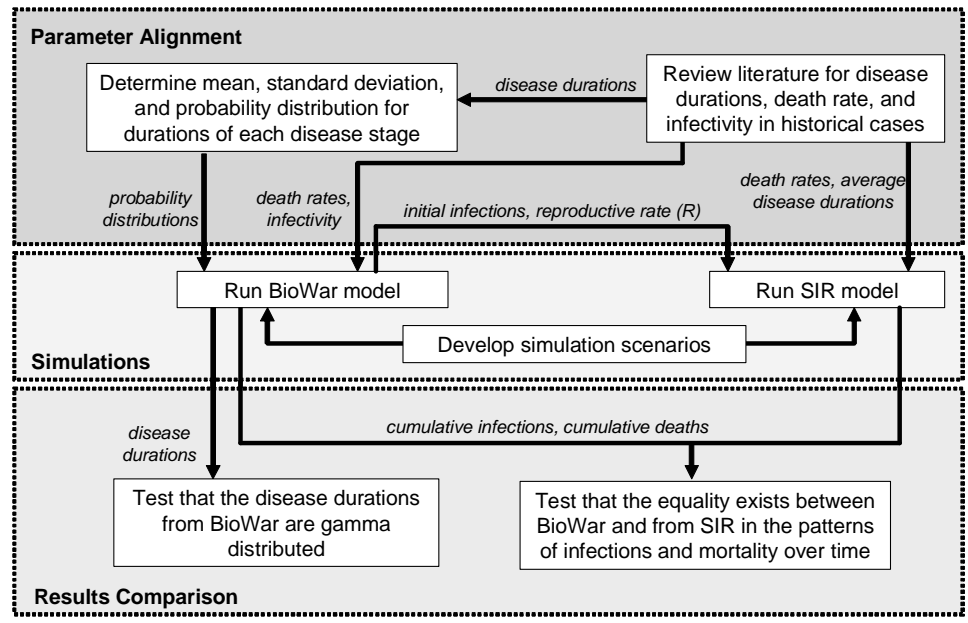

Fig 2. The process of model alignment 


\subsection{Parameter Alignment}

Although BioWar and SIR use are structurally very different, some of their model parameters are related. The parameter alignment process helped us to tune BioWar parameters to current epidemiology studies and to compare these parameters with those in the SIR model.

Both BioWar and SIR simulate disease progression in terms of the transition of infected individuals between disease stages, but with different stochastic framing. BioWar utilizes probability distributions to determine the duration of each disease stage for each infected agent. Based on statistical analyses of several empirical data sets [14], we model smallpox stage durations as gamma distributed with a mean $\mu$ and a standard deviation $\sigma[12,14-15,18,20]$. Table 1 lists the values of $\mu$ and $\sigma$ for the disease stages (incubation (I), prodrome (P), and fulminant. The fulminant stage is divided into fulminant-contagious (C) stage and fulminant-quarantined (Q)). In contrast, SIR uses transition probabilities to represent the rates sectors of a population move from one state to another. To align the SIR model with BioWar, we set the transition probabilities $^{1}$ to $(\mu)^{-1}$. Table 2 shows this parameterization for the SIR model based on the mean disease-stage durations from BioWar. Although we can conduct Monte Carlo simulations of the SIR model treating $\mu$ as a random variable of gamma distribution, the stochasticity is different from that in BioWar. In BioWar the gamma distribution describes the variation among individuals and in the SIR model it describes the variation around the population sector mean.

Table 1. Means and standard deviations for disease-stage durations of smallpox

\begin{tabular}{llll}
\hline & $\begin{array}{l}\text { State in } \\
\text { model }\end{array}$ & $\begin{array}{l}\text { SIR } \\
(\mu, \text { in days })\end{array}$ & $\begin{array}{l}\text { Standard deviation } \\
(\sigma, \text { in days })\end{array}$ \\
\hline incubation & I & 11.6 & 1.9 \\
prodromal & $\mathrm{P}$ & 2.49 & 0.88 \\
fulminant & $\mathrm{C}$ and Q & 16 & 2.83 \\
$\begin{array}{l}\text { contagious } \\
\begin{array}{l}\text { (without quarantine) } \\
\text { contagious }\end{array}\end{array}$ & $\mathrm{C}$ & 7 & 2.83 \\
\begin{tabular}{l} 
(with quarantine) \\
\hline
\end{tabular} & $\mathrm{C}$ & 2 & 1 \\
\hline
\end{tabular}

Table 2. Transition probabilities of the SIR model

\begin{tabular}{ll}
\hline Name of transition probability & Transition probability (in $[0,1]$ ) \\
\hline Leaving incubation $(\sigma)$ & $1 / 11.6$ \\
Leaving prodromal $(\alpha)$ & $1 / 2.49$ \\
Leaving contagious $(\gamma)$ & $1 / 7$ (without quarantine), $1 / 2$ (quarantine) \\
Leaving quarantine $(v)$ & $1 / 16$ \\
\hline
\end{tabular}

${ }^{1}$ In a Markov model, the transition probability from one state to another state is estimated by the inverse of the expected continuous duration of that state [21]. 
The disease transmission in the two models is also different stochastically. In BioWar, at a certain probability (infectivity), an infectious individual will infect other individuals whose physical distance is less than 100 meters from the infectious individual. As a result, the disease transmission probability (the number of new infections at a certain time) is determined by social factors influencing the interactions among agents, such as infectivity, social networks and their daily activities. In contrast, in SIR the disease transmission probability is equal to a transition probability of reproduction $(\beta)$ multiplied by the number of susceptible people plus the number of infectious people in the population. This transition probability is constant across the entire course of a simulation but the transmission probability is not.

Although we cannot align the two models stochastically, we can align the models at the same average level of disease transmission probability by using reproductive rates and the number of initial infections. Since BioWar can simulate the interactions among agents, reproductive rates are emergent properties (outputs) from simulations. Similarly, the number of initial infections is also an emergent property since BioWar can roughly estimate it from information about the location of an attack, the released amount of smallpox viruses, and the daily activities of the agents. In contrast, the SIR model cannot simulate the interactions so that it needs to determine $\beta$ and the number of initial infections before running the simulations. We experimentally derived both from BioWar experiments.

\subsection{Deriving Reproductive Rates from BioWar Experiments}

The reproductive rate $R$ is defined as the expected number of secondary cases produced by an infectious individual in a population of $S$ susceptible individuals. The basic reproductive rate $R_{0}$ represents the value of $R$ in a totally susceptible population $N$. When the natural birth rate and death rate are negligible compared to the transition probabilities, the expected reproduction rate $\mathrm{R}$ can be approximated as $\frac{\beta S}{\gamma}$ and $R_{0}$ is approximated as $\frac{\beta N}{\gamma}[1]$.

Based on the above definitions by Anderson and May, we experimentally calculated $R_{0}$ from BioWar outputs using equation (4). In this case, we can estimate $\beta=\frac{\not R_{0}}{N}$. This method of deriving $R_{0}$ has been used in another agent based simulation [14].

$$
R_{0}=\frac{\text { the number of secondary cases infected by initial infections }}{\text { the number of initial infections }} .
$$

Alternatively, we can also derive $\beta$ from BioWar directly. The number of new infections at certain time is equivalent to $\beta S C$ in the SIR model in which $S$ represents susceptible individuals and $C$ represents contagious population. Thus, $\beta$ at time $t$ can be approximated by (5). 


$$
\beta(t)=\frac{\text { new infections }(\mathrm{t})}{\text { susceptible }(\mathrm{t}) * \text { contagious }(\mathrm{t})} .
$$

Since BioWar is an agent based model, unlike SIR, the estimated transition probability is not a constant. In order to compare the average case in BioWar with SIR, we calculated $E(\beta)$ as the average of $\beta$ across time when it is larger than 0 ( $\beta=0$ means no new infections at the time). We can then estimate $R$ as (6).

$$
R=\frac{E(\beta) S}{\gamma} \text {. }
$$

\subsection{Simulations}

To compare the population level results from both BioWar and SIR, we simulated three smallpox attack scenarios: "base", "vaccination", and "quarantine". We started with a simplified base scenario and varied some of the parameters in other scenarios to increase the fidelity of the simulation. Table 3 lists the definitions of the three scenarios. For each scenario, we present the results as averages of 100 runs because the fluctuation of disease reproductive rates is negligible in around 100 runs.

We simulate an attack on the Washington, DC area, scaled down to $10 \%$ of its original size to speed up our simulations. The total population after scaling was about 55,900 . In the base scenario we assume the attack goes undetected and no public health responses or warnings occur after the attack. We assume that infected individuals are not contagious when they are in early-symptomatic stage because infectivity in this stage is considered to be negligible relative to the infectivity of later stages [14, 15]. All individuals in the city are assumed to be completely susceptible to smallpox in the base scenario.

Table 3. Simulation scenarios

\begin{tabular}{llll}
\hline Scenarios & $\begin{array}{l}\text { Residual immunity } \\
(\% \text { of total popula- } \\
\text { tion) }\end{array}$ & $\begin{array}{l}\text { Fresh vaccination } \\
(\% \text { of total popula- } \\
\text { tion) }\end{array}$ & $\begin{array}{l}\text { Is infected popula- } \\
\text { tion quarantined? }\end{array}$ \\
\hline base & $0 \%$ & $0 \%$ & no \\
vaccination & $46 \%$ & $50 \%$ & no \\
quarantine & $46 \%$ & $0 \%$ & $\begin{array}{l}\text { yes (on average, } 2 \\
\text { day after the onset } \\
\text { of rash) }\end{array}$ \\
\hline
\end{tabular}

We modeled an indoor smallpox attack where a random number of agents (less than 10) are initially infected. For the second and third scenario, we categorized the population based on their immunity: residual immunity, fresh vaccination, and no vaccina- 
tion. Agents with "residual immunity" 2 are assumed to have been vaccinated 30 or more years previously and their immunity against smallpox has weakened. In the US, $90 \%$ of the people born before 1972 were vaccinated, so about $50 \%$ of the contemporary population should have some level of the residual immunity [17]. In the scaled down DC population, approximately $46 \%$ (25,653 out of 55,930 people) were assigned residual immunity. Agents with "fresh vaccination" are assumed to have been vaccinated around two months before the attack. These individuals have high (but not perfect) immunity against smallpox. "No vaccination" means that the individuals had never been vaccinated. Table 4 lists the assumed probability of death following infection and infectivity for each of the three immune status categories [5, 9, 20].

Both "vaccination" and "quarantine" scenarios consider the residual immunity of the population. In addition, the "vaccination" scenario examines the effects of fresh vaccination among the population and the "quarantine" scenario examines the effects of infectious individuals being quarantined in around 2 days after the onset of rash so they will not infect other agents. In the "vaccination" scenario, agents are randomly selected for vaccination and agents who had been vaccinated before 1972 may be vaccinated again.

Table 4. Simulation parameters for different population categories

\begin{tabular}{|c|c|c|c|}
\hline & $\begin{array}{l}\text { Residual im- } \\
\text { munity }\end{array}$ & $\begin{array}{l}\text { Fresh vaccina- } \\
\text { tion }\end{array}$ & No vaccination \\
\hline Infectivity & $50 \%$ & $5 \%$ & $95 \%$ \\
\hline $\begin{array}{l}\text { Probability of death fol- } \\
\text { lowing infection }\end{array}$ & $7 \%$ & $2 \%$ & $30 \%$ \\
\hline
\end{tabular}

\section{Results and Discussion}

We conducted both qualitative graph comparisons and statistical tests on the population level results. For each set of results from BioWar and SIR, we first compared them graphically and then statistically. For the disease-stage durations, we conducted parametric chi-square $\left(X^{2}\right)$ tests to see if BioWar results are gamma distributed. To compare the rate of transmission and mortality from smallpox over time, we used non-parametric two sample hypothesis tests to compare the data generated by the two models.

\subsection{Disease-Stage Durations}

BioWar smallpox stage (incubation, prodrome, fulminant) durations are modeled as gamma distributed while SIR disease-stage durations are the average case of the gamma distributions. The average of the individual stage durations generated by

\footnotetext{
${ }^{2}$ Here we refer to individuals who were vaccinated many years ago in contrast with fresh vaccination. However, this term is usually used to describe all individuals who have been vaccinated.
} 
BioWar should be close to the durations the infected population spends in each disease-stage in SIR. To verify this, we tested if the BioWar disease-stage durations are actually gamma distributed. The point of testing is simply to verify that BioWar is doing what it is told to do. In agent-based simulations, this should not be taken for granted.

We calculated the three disease-stage durations for 1000 infected agents in BioWar. Graphically, Fig. 3 shows that the BioWar distribution of duration of the incubation period is similar to the gamma distribution and to literature values [15]. However, $X^{2}$ tests rejected the hypothesis that the incubation period is gamma distributed ( $\mathrm{p}$ value $>0.05)$, but could not reject this hypothesis for the prodrome and fulminant stages (Table 5).

The prodrome and fulminant stage durations simulated in BioWar are gamma distributed. The distribution of the incubation stage (Fig. 3) resembles the gamma distribution, but is too peaked.

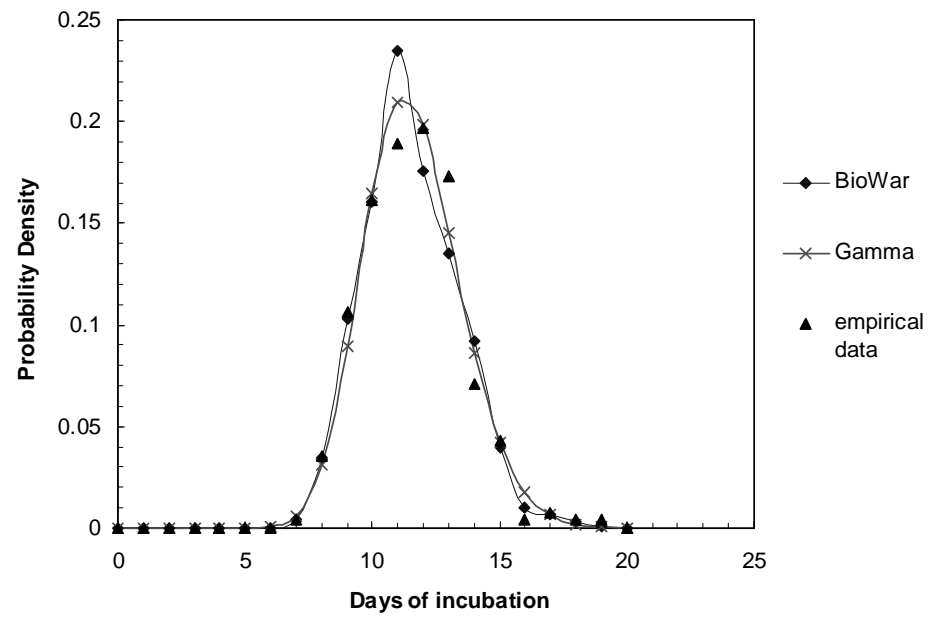

Fig 3. A comparison of distribution of the incubation stage duration in BioWar with the theoretical [14] and empirical [15] data

Table 5. Goodness-of-fit test for smallpox stage durations of BioWar. ** Gamma distributed, significant at $\alpha>0.05$

\begin{tabular}{cccc}
\hline Disease stage & $X^{2}$ & Degree of freedom & P-value \\
\hline incubation & 17.75 & 9 & 0.04 \\
prodromal $^{* *}$ & 8.95 & 5 & 0.11 \\
fulminant $^{* *}$ & 19.89 & 13 & 0.10 \\
\hline
\end{tabular}




\subsection{Infection and Mortality}

We aligned the transition probability of reproduction $(\beta)$ of SIR using reproductive rate $R$ generated from BioWar, shown in Table 6. Table 7 displays BioWar and SIR estimations for the three scenarios. The difference in total mortality among infected individuals from the two models is less than $1 \%$ in all three scenarios. As illustrated in Figures $4 \mathrm{a}-4 \mathrm{c}$, the progression of infection in the BioWar and SIR models are qualitatively similar. We obtained similar results from graph comparisons on overtime mortality.

We conducted nonparametric two-sample hypothesis tests to statistically compare the patterns of infection and mortality from the two models over time. Using the PetoPeto-Prentice test [11], we tested the hypothesis that the over-time infection data from the BioWar and SIR models are statistically equivalent, in the sense that they could be generated from the same population with a unique underlying over-time pattern of infection. The Peto-Peto-Prentice test estimates expected numbers of infections at each time point using the combined output from the BioWar and SIR models, under the null hypothesis that there is no difference between the over-time patterns of infection in the two groups. The expected values are compared to the observed number of infections predicted by each model at each time point. These differences are combined into a single global statistic, which has a $X^{2}$ distribution with 1 degree of freedom (for the test, $\mathrm{df}=$ number of groups compared -1 ). The same test is used to compare the mortality patterns in the BioWar and SIR models.

Table 6. Reproductive rates estimated from BioWar for three scenarious and three population categories.

\begin{tabular}{llccc}
\hline Scenario & reproductive rate & $\begin{array}{c}\text { no vacci- } \\
\text { nation }\end{array}$ & $\begin{array}{c}\text { residual } \\
\text { immunity }\end{array}$ & $\begin{array}{c}\text { fresh } \\
\text { nated }\end{array}$ \\
\hline base & $\mathrm{R}_{0}$ & 4.92 & N.A. & N.A. \\
vaccination & $\mathrm{R}$ & 3.86 & N.A. & N.A. \\
& $\mathrm{R}_{0}$ & 2.13 & 1.28 & 0.44 \\
quarantine & $\mathrm{R}$ & 1.31 & 0.53 & 0.20 \\
& $\mathrm{R}_{0}$ & 1.84 & 1.45 & N.A. \\
& $\mathrm{R}$ & 1.17 & 0.38 & N.A. \\
\hline
\end{tabular}

Table 7. A comparison of BioWar and SIR average results for the three scenarios

\begin{tabular}{llcccc}
\hline Scenario & Model & $\begin{array}{l}\text { Initial } \\
\text { Infections }\end{array}$ & $\begin{array}{l}\text { Cumulative } \\
\text { Infections }\end{array}$ & $\begin{array}{l}\text { Cumulative } \\
\text { Deaths }\end{array}$ & $\begin{array}{l}\text { Mortality among } \\
\text { Infections }\end{array}$ \\
\hline base & SIR & 7 & 54,765 & 16,851 & $31 \%$ \\
& BioWar & 7 & 54,345 & 16,724 & $31 \%$ \\
vaccina- & SIR & 6 & 27,262 & 4876 & $18 \%$ \\
tion & BioWar & 6 & 25,766 & 4748 & $18 \%$ \\
quaran- & SIR & 5 & 30,119 & 7008 & $23 \%$ \\
tine & BioWar & 5 & 27,815 & 6597 & $24 \%$ \\
\hline
\end{tabular}


The results for our three scenarios are shown in Tables $8 \mathrm{a}$ and $8 \mathrm{~b}$. A large $X^{2}$ (and correspondingly small p-value) indicates a statistically detectable difference between the output generated by the BioWar and SIR models. Note that the total number of infections or deaths in the BioWar and SIR output combined roughly reflects the amount of data available to the test. Even a small difference between infection or mortality curves may be detected with large amounts of data.

A statistically significant difference between over-time infection was detected in all scenarios ( $p$-value < 0.05 ). The test shows that the models are in better agreement in regards to cumulative mortality, at least in the base case and vaccination scenario. For these, the test was unable to reject the hypothesis of equality for the two time series.. While the Peto-Peto-Prentice test cannot prove equivalence between the BioWar and SIR mortality results in "base" and "vaccination" scenarios, the fact that it was unable to detect a significant difference supports our qualitative conclusion that the patterns of smallpox deaths in the two models are similar, though not identical.

Table 8a. Results of Peto-Peto-Prentice tests for BioWar and SIR estimates on cumulative infections. Number of infections refer to the combined infections resulting from the BioWar and the SIR model

\begin{tabular}{lcccc}
\hline Scenario & $\begin{array}{l}X^{2} \\
(\text { degree } \\
\text { dom }=1)\end{array}$ & $\begin{array}{c}\text { P- } \\
\text { free- }\end{array}$ & $\begin{array}{c}\text { Time se- } \\
\text { value } \\
\text { tions infec- }\end{array}$ & $\begin{array}{c}\text { Number of } \\
\text { infections }\end{array}$ \\
\hline base & 113.03 & $<0.001$ & Different & 109,096 \\
\hline vaccination & 4.08 & 0.0434 & Different & 53,016 \\
\hline quarantine & 233.82 & $<0.001$ & Different & 57,924 \\
\hline
\end{tabular}

Table 8b. Results of Peto-Peto-Prentice tests for BioWar and SIR estimates on cumulative deaths. Number of deaths refer to the combined deaths resulting from the BioWar and the SIR model

\begin{tabular}{lcclc}
\hline Scenario & $\begin{array}{c}X^{2} \\
(\text { degree of free- } \\
\text { dom=1) }\end{array}$ & P-value & $\begin{array}{c}\text { Time series } \\
\text { of deaths }\end{array}$ & $\begin{array}{c}\text { Number } \\
\text { of deaths }\end{array}$ \\
\hline base & 0.59 & 0.4438 & Same & 33,575 \\
\hline vaccination & 0.6 & 0.4369 & Same & 9,624 \\
\hline quarantine & 15.45 & 0.0001 & Different & 13,605 \\
\hline
\end{tabular}




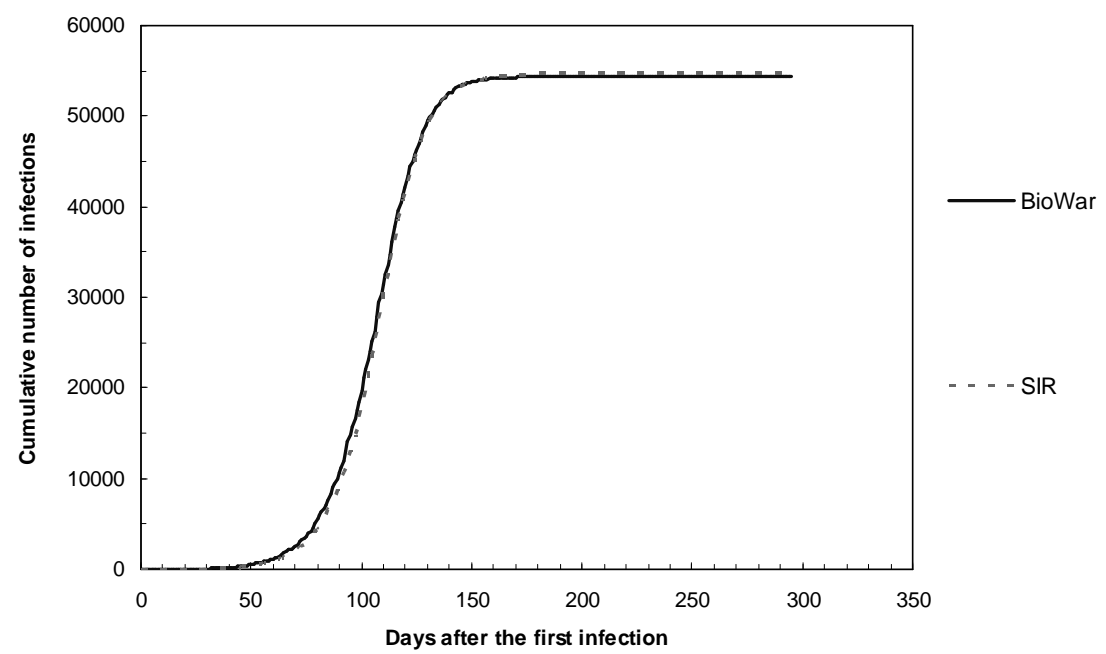

Fig 4a. The comparison of BioWar and SIR in cumulative infections ("base" scenario)

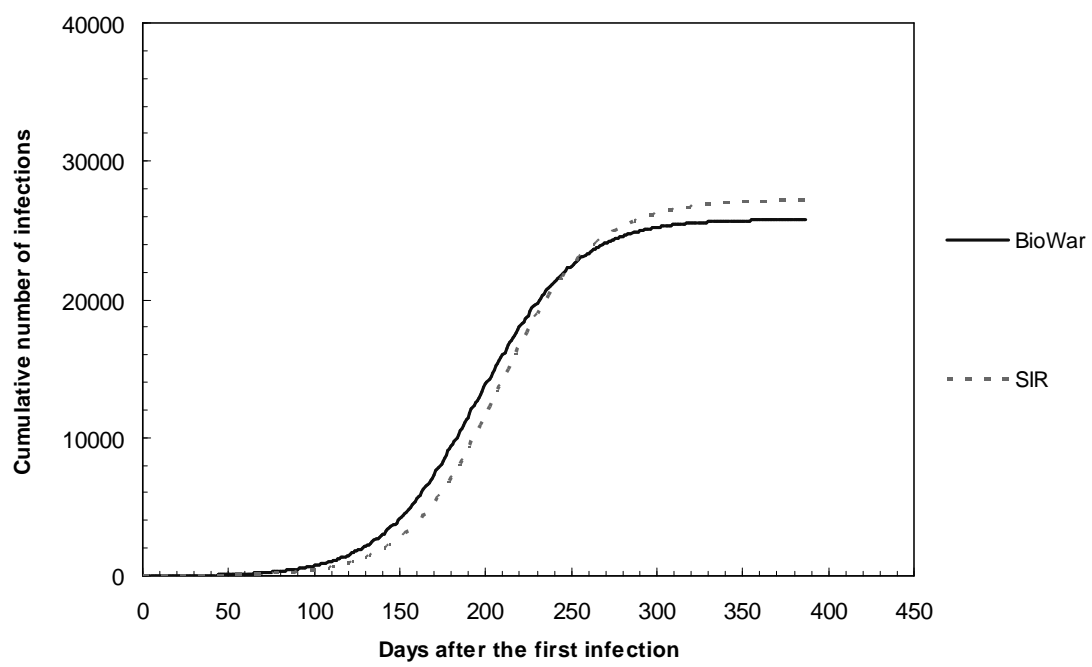

Fig 4 b. The comparison of BioWar and SIR in cumulative infections ("vaccination" scenario) 


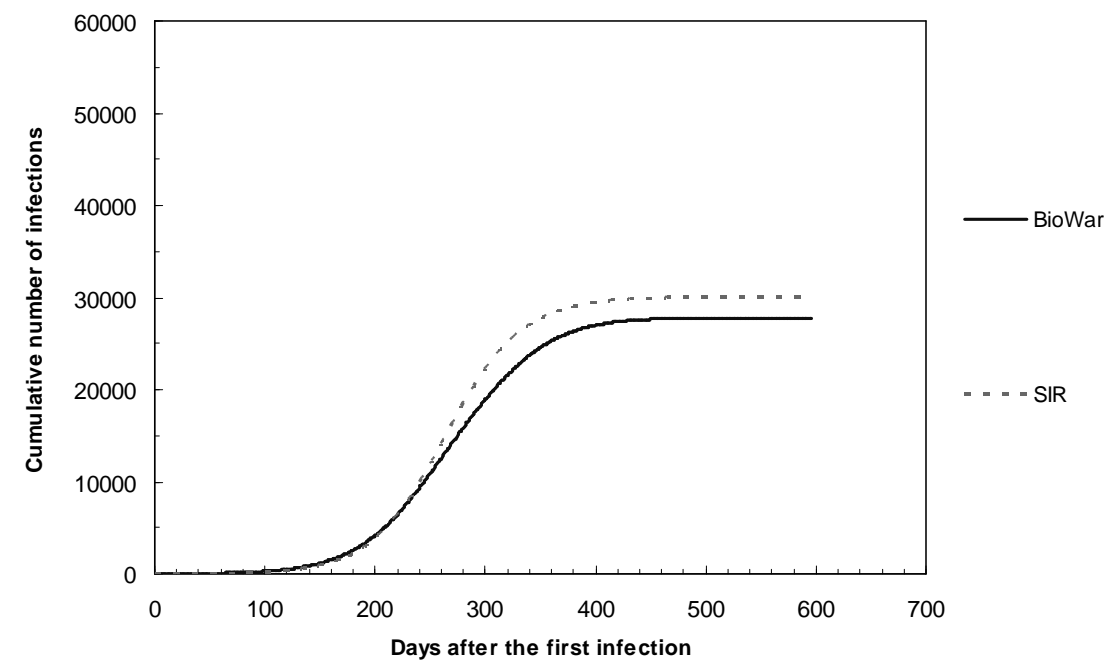

Fig 4c. The comparison of BioWar and SIR in cumulative infections ("quarantine" scenario)

\subsection{Discussion}

Using smallpox attack simulations, we developed a methodology for comparing the BioWar agent-based model to the equivalent SIR model for contagious disease outbreaks. On a gross level such models should give approximately the same results, but subtle differences should exist because of the differences in mixing assumptions. This was the outcome of the docking, and serves as a partial validation of BioWar, demonstrating that it is at least able to produce fairly similar results to the accepted standard epidemiological model. The differences between BioWar and SIR were most evident in the scenarios involving vaccination and quarantine. It would be expected that the agent-based model would produce different results here, as the agent-level complexity required for such scenarios is easily accommodated by BioWar, but not by SIR.

The main benefit of validating the disease progression process separately from the disease transmission process is to clarify the sources of discrepancies in the simulations. We detected a deviation from expected incubation-duration distribution in BioWar which may have contributed to the differences found in model outputs.

Only certain aspects of the models could be compared. Because of the different ways the models account for parameter uncertainty, it is necessary to compare average results over numerous runs. We found that $R_{0}$ (average number of secondary cases in a totally susceptible population infected by one primary case) commonly used in SIR model, is not comparable to $R_{0}$ in BioWar. In BioWar, $R_{0}$ changes each run. $R$ (the reproduction rate over the entire simulation) is different from $R_{0}$ and is calculated as an average reproduction rate over all relevant time steps in a simulation. However, no distinction between $R$ and $R_{0}$ is made in SIR and $R$ is constant for each run and at each simulation step. This finding implies that, when comparing an agent-based model and 
the SIR model, modelers should align $R_{0}$ (or $R$ ) in the SIR with $R$ in the agent-based model since only the average cases are comparable. Aligning $R_{0}$ in SIR with $R_{0}$ in an agent-based model will provide a misleading comparison.

When the level of detail in a simulation increases, the number of model parameters needed increases. For example, the transmission probability may vary by age group or occupation (such as medical workers, family members of an infected person, or general public). BioWar provides a way to manage these model parameters in order to represent the heterogeneous properties of individuals. Although we can revise SIR model to simulate the same level of fidelity by dividing the population into several categories, it is not advisable because the number of model parameters would increase nonlinearly to an unmanageable level. In addition, revising SIR to have finer population categories overlooks an important aspect of disease transmission: the fact that the population reproductive rate is actually partly the result of interactions between individuals and these interactions are emergent properties of agent-based models which cannot be generated from the SIR model.

\section{Conclusions}

We developed a methodology to align a multi-agent model of weaponized biological attacks, BioWar, with the classical susceptible-infected-recovered (SIR) model. Using smallpox attack simulations, we showed that average results from BioWar are comparable to the SIR model, when the models are properly parameterized. The key parameters include the average disease-stage durations, the reproductive rate, the initial infection and the probability of death following infection.

The successful docking of the two radically different models provided a degree of confidence in the agent-based model, showing that its results are not far from those of the established SIR model. This work is our first step of the larger task on validating BioWar. Tools for finer-granularity validation of agent-based models are underway [36]. Based on this foundation, we will further investigate the policy responses against the outbreaks of contagious diseases by changing heterogeneous properties of agents (such as social networks, daily activities, and reactions to an attack), which cannot be simulated in a SIR model.

The differences in model inputs of smallpox simulations may lead to a different result [30]. It is important for policy makers to understand the differences and similarities between agent-based models and the SIR model before making decisions based on any one model. It is also important for modelers to realize what model inputs and outputs are comparable between the two models. We expect our results will help policy makers and other modelers.

\section{Acknowledgements:}

The authors would like to thank Neal Altman and Démian Nave for their support on this paper. This research was supported, in part, by DARPA for work on Scalable Biosurveillance Systems, the NSF IGERT9972762 in CASOS, the MacArthur Foun- 
dation, and by the Carnegie Mellon Center on Computational Analysis of Social and Organizational Systems. The computations were performed on the National Science Foundation Terascale Computing System at the Pittsburgh Supercomputing Center. Any opinions, findings, conclusions or recommendations expressed in this material are those of the authors and do not necessarily reflect the views of DARPA, the National Science Foundation, the Pittsburgh Supercomputing Center, the MacArthur Foundation, or the US Government.

\section{References}

1. Anderson, R.M. and May, R.M.: Directly transmitted infections diseases: control by vaccination. Science. Vol., 215 (1982) 1053-1060.

2. Anderson, R.M. and May, R.M.: Infectious Diseases in Humans: Oxford University Press (1992).

3. Axtell, R., Axelrod, R., Epstein, J.M., and Cohon, M.D.: Aligning simulation models: a case study and results. Computational and Mathematical Organization Theory. Vol. 1, (1996).

4. Bailey, N.J.T.: The Mathematical Theory of Infectious Diseases and Its Applications. 2nd ed. New York: Oxford University Press (1975).

5. Bartlett, J., Borio, L., and et. al: Smallpox Vaccination in 2003: Key Information for Clinicians. Clinical Infectious Diseases. Vol. 36, (2003) 883-902.

6. Bozzette, S.A., Boer, R., Bhatnagar, V., Brower, J.L., Keeler, E.B., Morton, S.C., and Stoto, M.A.: A model for a smallpox-vaccination policy. New England Journal of Medicine. Vol., 348 (2003) 416-425.

7. Carley, K., Altman, N., Kaminsky, B., Nave, D., and Yahja, A.: BioWar: A City-Scale Multi-Agent Network Model of Weaponized Biological Attacks, Technical Report (CMUISRI-04-101). Pittsburgh, PA: CASOS, Carnegie Mellon University (2004), http://reportsarchive.adm.cs.cmu.edu/isri2004.html.

8. CDC: Smallpox fact sheet: smallpox overview. Centers for Disease Control and Prevention (2002). http://www.bt.cdc.gov/agent/smallpox/overview/disease-facts.asp.

9. CDC: Smallpox fact sheet: vaccine overview. Centers for Disease Control and Prevention (2003). http://www.bt.cdc.gov/agent/smallpox/vaccination/facts.asp.

10.Chen, L.-C., Carley, K.M., Fridsma, D., Kaminsky, B., and Yahja, A.: Model alignment of anthrax attack simulations. CASOS working paper, Carnegie Mellon University (2003).

11.Cleves, M.A., Gould, W.W., and Gutierrez, R.G.: An Introduction to Survival Analysis using Stata: Stata Press (2002).

12.Creighton, C.: A History of Epidemics in Britain. Vol. 2. Cambridge: Cambridge Univ. Press (1891).

13.Diekmann, O. and Heesterbeek, J.A.P.: Mathematical Epidemiology of Infectious Diseases: Model Building, Analysis and Interpretation. New York: John Wiley \& Sons (2000).

14.Eichner, M. and Dietz, K.: Transmission potential of smallpox: Estimates based on detailed data from an outbreak. American Journal of Epidemiology. 158 (2003) 110-117.

15.Fenner, F., Henderson, D.A., Arita, I., Jezek, Z., and Ladnyi, I.D.: Smallpox and its Eradication. Geneva: WHO (1988).

16.Ferguson, N.M., Keeling, M.J., Edmunds, W.J., Gani, R., Grenfell, B.T., Anderson, R.M., and Leach, S.: Planning for smallpox outbreaks. Nature. 425 (2003) 681-685.

17.Gani, R. and Leach, S.: Transmission potential of smallpox in contemporary populations. Nature. 414 (2001) 748-751.

18.Gelfand, H.M. and Posche, J.: The recent outbreak of smallpox in Meschede, West Germany. American Journal of Epidemiology. Vol. 93, 4 (1971). 
19.Halloran, M.E., Longini, I.M., Nizam, A., and Yang, Y.: Containing bioterrorist smallpox. Science. 298 (2002) 1428-1432.

20.Hammarlund, E., Lewis, M.W., Hansen, S.G., Strelow, L.I., Nelosn, J.A., Sexton, G.J., Hanifin, J.M., and Slifka, M.K.: Duration of antiviral immunity after smallpox vaccination. Nature Medicine,. Vol. 9, 9 (2003) 1131-1137.

21.Henderson, D.A., Inglesby, and et al.: Smallpox as a Biological Weapon - Medical and Public Health Management. Journal of the American Medical Association. Vol. 281, 22 (1999) 2127-2137.

22.Henderson, D.A.: Smallpox: public health threat. Encyclopedia of Life Sciences. Elsevier (2000).

23.Henderson, D.A.: Smallpox. in Encyclopedia of Microbiology (2000).

24.Kaplan, E.H., Craft, D.L., and Wein, L.M.: Emergency response to a smallpox attack: The case for mass vaccination. Proceedings of National Academy of Science USA. 99 (2002) 10935-10940.

25.Mack, T.: Smallpox in Europe, 1950-1971. Journal of Infectious Disease. 125 (1972) 161169.

26.Meltzer, M.I., Damon, I., LeDuc, J.W., and Millar, J.D.: Modeling potential responses to smallpox as a bioterrorist weapon. Emerging Infectious Disease. 7 (2001) 959-969.

27.NDSC: Biological threat agents. National Disease Surveillance Centre (2003).

28.O'Toole, T. and Inglesby, T.V.: Facing the Biological Weapons Threat. The Lancet. Vol. 357 (Editorial), (2001).

29.Patrick, W.C.: Biological Terrorism and Aerosol Dissemination. Politics and the Life Sciences. Vol. 15, (1996) 208-210.

30.Powell, K.: Models call for vaccinations before bioterror attack. Nature. 15 Nov. (2002).

31.Rabiner, L.R.: A tutorial on hidden Markov models and selected applications in speed recognition. Proceedings of the IEEE. 2 (1989) 257-268.

32.Sargent, R.G.: Simulation Model Validation. in Simulation and Model-Based Methodologies: An Integrative View, T.I. Oren, B.P. Zeigler, and M.S. Elzas: Editors. Springer- Verlag: Heidelberg, Germany (1984) 537-555.

33.Sargent, R.G.: Verification and validation of simulation models. in Proceedings of the 2003 Winter Simulation Conference (2003).

34.Schlesinger, S., Crosbie, R.E., Gagne, R.E., Innis, G.S., Lalwani, C.S., Loch, J., Sylvester, R.J., Wright, R.D., Kheir, N., and Bartos, D.: Terminology for model credibility. Simulation. Vol. 32, 3 (1979) 103-104.

35.UPMC: Smallpox Fact Sheet. Center for Biosecurity, University of Pittsburgh Medical Center (2003). http://www.upmc-biosecurity.org/pages/agents/smallpox_facts.html.

36.Yahja, A.: WIZER: Automated Validation of Large-Scale Multi-Agent Systems. CASOS, Carnegie Mellon University (2004). 